\title{
Corrigendum to "Massive Acetaminophen Overdose Treated Successfully with N-Acetylcysteine, Fomepizole, and Hemodialysis"
}

\author{
Michael H. Chiu, ${ }^{1}$ Natalia Jaworska, ${ }^{1}$ Nicholas L. Li, ${ }^{2}$ and Mark Yarema ${ }^{1,3,4}$ \\ ${ }^{1}$ Department of Critical Care Medicine, Cumming School of Medicine, University of Calgary, AB, Canada \\ ${ }^{2}$ Department of Medicine, Division of Nephrology, Cumming School of Medicine, University of Calgary, AB, Canada \\ ${ }^{3}$ Poison and Drug Information Service, Alberta Health Services, Calgary, AB, Canada \\ ${ }^{4}$ Department of Emergency Medicine, University of Calgary, Calgary, AB, Canada
}

Correspondence should be addressed to Michael H. Chiu; michael.chiu@albertahealthservices.ca

Received 1 November 2021; Accepted 1 November 2021; Published 17 November 2021

Copyright (c) 2021 Michael H. Chiu et al. This is an open access article distributed under the Creative Commons Attribution License, which permits unrestricted use, distribution, and reproduction in any medium, provided the original work is properly cited.

In the article titled "Massive Acetaminophen Overdose Treated Successfully with N-Acetylcysteine, Fomepizole, and Hemodialysis" [1], there was an error in Figure 1(a), where the unit of acetaminophen in the $y$-axis should be corrected to " $\mu \mathrm{mol} / \mathrm{L}$."

The authors confirm that this does not affect the results and conclusions of the article.

\section{References}

[1] M. H. Chiu, N. Jaworska, N. L. Li, and M. Yarema, "Massive Acetaminophen Overdose Treated Successfully with N-Acetylcysteine, Fomepizole, and Hemodialysis," Case Reports in Critical Care, vol. 2021, Article ID 6695967, 5 pages, 2021. 